\title{
Integration of High Performance Silicon Optical Modulators
}

\author{
D. J. Thomson ${ }^{1}$, F. Y. Gardes ${ }^{1}$, Y. Hu ${ }^{1}$, G. Mashanovich ${ }^{1}$, G. T. Reed ${ }^{1}$, L. Zimmermann ${ }^{2}$, D. Knoll ${ }^{2}$, S. Lischke ${ }^{2}$, H. Porte ${ }^{3}$, B. \\ Goll $^{4}$, H. Zimmermann ${ }^{4}$, L. Ke ${ }^{5}$, P. Wilson ${ }^{5}$, S-W. Chen ${ }^{6}$, S. H. Hsu ${ }^{6}$, G.-H. Duan ${ }^{7}$, A. Le Liepvre ${ }^{7}$, C. Jany ${ }^{7}$, A.Accard ${ }^{7}$, M. \\ Lamponi $^{7}$, D. Make ${ }^{7}$, F. Lelarge ${ }^{7}$, S. Messaoudene ${ }^{8}$, D. Bordel ${ }^{8}$, and J.-M. Fedeli ${ }^{8}$, S. Keyvaninia ${ }^{9}$, G. Roelkens ${ }^{9}$ and D. Van \\ Thourhout $^{9}$
}

\author{
${ }^{1}$ Optoelectronics Research Centre, University of Southampton, Southampton, Hampshire, SO17 1BJ, UK \\ ${ }^{2}$ IHP, Im Technologiepark 25, 15236 Frankfurt (Oder), Germany \\ ${ }^{3}$ PHOTLINE Technologies, ZI Les Tilleroyes - Trépillot, 16 rue Auguste Jouchoux, 25000 Besançon \\ ${ }^{4}$ Vienna University of Technology, EMCE, Gußhausstr. 25/354, A-1040 Vienna, Austria \\ ${ }^{5}$ School of electronics and computer science, University of Southampton, Southampton, Hampshire, SO17 1BJ, UK \\ ${ }^{6}$ High-Speed Devices and Integrated Circuits Group, National Tsing Hua University, Hsinchu, Taiwan \\ ${ }^{7}$ III-V Lab, a joint lab of 'Alcatel-Lucent Bell Labs France', 'Thales Research and Technology' and 'CEA Leti', Campus \\ Polytechnique, 1, Avenue A. Fresnel, 91767 Palaiseau cedex, France. \\ ${ }^{8}$ CEA LETI, Minatec, 17 rue des Martyrs, F-38054 GRENOBLE cedex 9, France. \\ ${ }^{9}$ Photonics Research Group, INTEC, Ghent University-IMEC, Sint-Pietersnieuwstraat 41, B-9000 Ghent, Belgium
}

\begin{abstract}
We present our recent work on high speed silicon optical modulators developed within the UK silicon photonics and HELIOS projects. Examples of their integration with other photonic and electronic elements is also presented.
\end{abstract}

Keywords-Optical modulator, Mach Zehnder, Silicon-oninsulator, Integrated Optics, Electro-optic modulation, integration, Laser, CMOS, BiCMOS.

\section{INTRODUCTION}

$\mathrm{H}$ IGH performance optical modulators are essential for the development of a range of integrated optical circuits with different functionality. The previous decade has seen a period of dramatic improvements in performance of silicon optical modulators and silicon based photonic components as a whole. The integration of these different components as well as electronic elements is the next challenge which is being overcome. In this paper we begin by presenting the different modulator designs which we have been working on within the UK Silicon Photonics (UKSP) and HELIOS projects as well as the results obtained from these structures. We then overview the integration of these modulators with different electronic and photonic elements.

\section{MODUlATOR DESIGNS}

Within the UKSP and HELIOS projects we have been developing different designs of high performance carrier depletion optical modulators. Within both projects the designs have been based around the concept of self-aligned fabrication $[1,2]$ to relax the fabrication tolerances that are acceptable and therefore provide the potential for a reliable performance, high yield and lower cost production. The first design, shown in figure 1 , is based in silicon-on-insulator (SOI) with a $220 \mathrm{~nm}$ thick overlayer. The rib section of the waveguide $(220 \mathrm{~nm} x$ $400 \mathrm{~nm})$ and slab (100nm thick) to one side is doped $\mathrm{p}$ type $\left(3 \mathrm{e} 17 . \mathrm{cm}^{-3}\right)$ and the slab to the other side doped $\mathrm{n}$ type $\left(1.5 \mathrm{e} 18 . \mathrm{cm}^{-3}\right)$. The higher doping density of the $\mathrm{n}$ type region ensures that depletion region extends mainly into the waveguide during modulation. Highly doped $\mathrm{p}$ and $\mathrm{n}$ type regions are positioned away from the waveguide rib in the slabs to form ohmic contacts with coplanar waveguide electrodes which are used to drive the device at high speed.

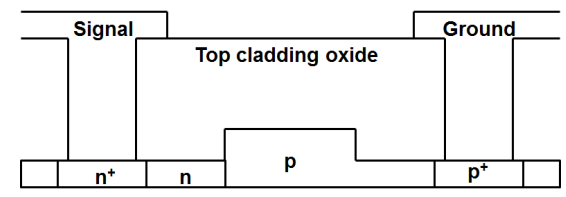

Buried oxide

Fig. 1. Cross sectional diagram of the $220 \mathrm{~nm}$ carrier depletion phase modulator. Note that the silicon substrate is omitted from the diagram.

Operation at $10 \mathrm{Gbit} / \mathrm{s}$ with a $18 \mathrm{~dB}$ extinction ratio, $40 \mathrm{Gbit} / \mathrm{s}$ with a $10 \mathrm{~dB}$ extinction ratio and $50 \mathrm{Gbit} / \mathrm{s}$ with a $3 \mathrm{~dB}$ extinction ratio is demonstrated when slightly different variants are incorporated in Mach Zehnder Interferometer (MZI) structures [1,3]. A ring resonator version is also demonstrated with operation up to $40 \mathrm{Gbit} / \mathrm{s}$ [4].

The second variant of phase modulator is shown in figure 2 . This version is based in $400 \mathrm{~nm}$ thick overlayer SOI which allows for waveguides to be produced which can support the propagation of both TE and TM modes. Depletion is achieved at a pn junction which wraps around the top and two sides of the waveguide rib. The purpose of this design is to achieve polarisation independent modulation. This can be obtained by tailoring the position of the doped regions and the density of active dopants within them to match the modulation efficiency for both the TE and TM polarisation modes. The $\mathrm{p}$ and $\mathrm{n}$ type 
regions are both doped to a level of $\sim 1 \mathrm{e} 18 . \mathrm{cm}^{-3}$. Operation at $10 \mathrm{Gbit} / \mathrm{s}$ and $40 \mathrm{Gbit} / \mathrm{s}$ with the same extinction ratio for both TE and TM polarisations is demonstrated [2].

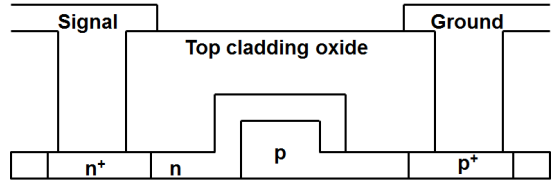

Buried oxide

Fig. 2. Cross sectional diagram of the $400 \mathrm{~nm}$ carrier depletion phase modulator. Note again that the silicon substrate is omitted from the diagram.

\section{MODULATOR INTEGRATION}

The next stage for optical modulators is the integration with other photonic and electronic components to form functional circuits. This section will overview some examples of the integration of these silicon optical modulators. Within the UKSP project a Mach Zehnder modulator (MZM) carrier depletion silicon optical modulator has been integrated with CMOS driving electronics using a wire bonding approach. An optical microscope image of this is shown in the left hand image of figure 3. The MZI used is asymmetric and has $3.5 \mathrm{~mm}$ phase modulators in either arm. Multimode mode interference (MMI) structures are used to split and recombine the light in the MZI arms. The dual drive CMOS driver was fabrication using the IBM-8RF $130 \mathrm{~nm}$ process [5]. Operation at $10 \mathrm{Gbit} / \mathrm{s}$ as targeted by the design of the driver is demonstrated.

Also within UKSP a full single channel link with transmitter and receiver integrated electronics is demonstrated. The TIA was fabricated using $180 \mathrm{~nm}$ TSMC technology [6]. An optical microscope image of their integration is shown in figure 3 (right).
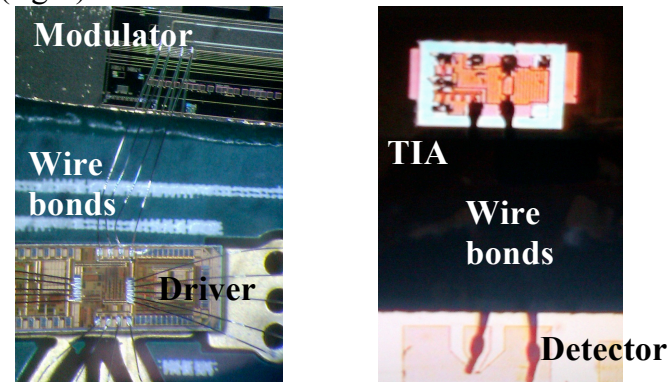

Fig. 3. Optical microscope image of the wire bonded carrier depletion modulator and CMOS driver (left) and photodetector and TIA (right).

Within the HELIOS project partners have collaborated to provide the first demonstration of frontend integration of a silicon photonics modulator with BiCMOS. The $220 \mathrm{~nm}$ carrier depletion modulator as shown in figure 1 has been integrated with a dual drive SiGe RF driver amplifier in the same silicon chip. An optical microscope image of the device under test is shown in figure 4. The Mach-Zehnder interferometer (MZI) is symmetrical and contains $2 \mathrm{~mm}$ phase modulators in either arm. Multimode interference structures are used to combine and split the light to the two MZI waveguide arms. A DC tuning section comprising low speed carrier depletion modulators is included to allow tuning of the MZI operating point. The target data rate of $10 \mathrm{Gbit} / \mathrm{s}$ is achieved with an extinction ratio in excess of $8 \mathrm{~dB}$.

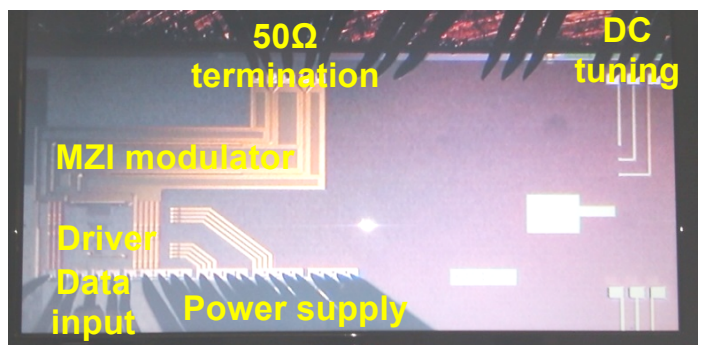

Fig. 4. Annotated optical microscope image of the frontend integrated BiCMOS driver and carrier depletion modulator during testing.

Also within the HELIOS project a tunable transmitter, integrating a hybrid III-V/Si laser fabricated by wafer bonding and the carrier depletion based silicon MZM is demonstrated [7]. In this case an asymmetric MZI with $3 \mathrm{~mm}$ phase modulators was used. 10Gbit/s operation was demonstrated with an extinction ratio between 6 and 10dB. A BER better than $1 \mathrm{e}-9$ is achieved.

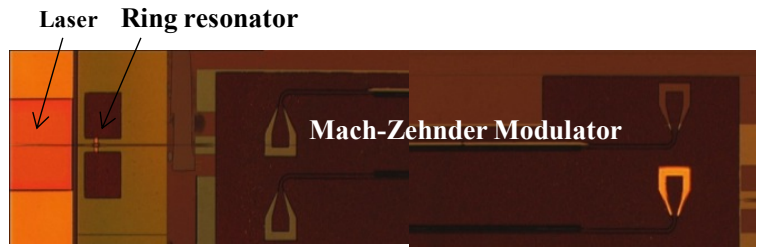

Fig. 5. Optical microscope image of the III-V source and carrier depletion modulator integration.

\section{ACKNOWLEDGEMENTS}

Authors acknowledge funding by the European Commission under project HELIOS (pHotonics Electronics functional Integration on CMOS), FP7-224312. D.J.T, F.Y.G and G.T.R acknowledge funding from the EPSRC in the UK to support the UK Silicon Photonics project.

\section{REFERENCES}

[1] D. J. Thomson et al., "High contrast 40Gbit/s optical modulation in silicon," Opt. Express, 19(12), 11507 - 11516, (2011).

[2] F. Y. Gardes et al., " $40 \mathrm{~Gb} / \mathrm{s}$ silicon photonics modulator for TE and TM polarisations," Opt. Express, 19(12), pp. 11804-11814, (2011).

[3] D. J. Thomson et al., "50-Gb/s silicon optical modulator," IEEE Photon. Technol. Lett 24(4), 234-236 (2012).

[4] D. J. Thomson et al., "Self-aligned silicon ring resonator optical modulator with focused ion beam correction," J. Opt. Soc. Am. B, 30(2), $445-449,(2012)$.

[5] K. Li and P. Wilson, "An improved push-pull driver using $0.13 \mu \mathrm{m}$ CMOS" In: 2013 IEEE International Symposium on Circuits and Systems, ISCAS, (2013).

[6] S. H. Hsu et al., "CMOS Broadband Amplifiers for Optical Communications and Optical Interconnects," IEEE Symposium of RF Integration Technology, (2011).

[7] G.-H. Duan et al. "Integrated III-V/Si Laser and Transmitter," IPRM 2012 , pp. 27-30, 2012) 